\title{
Emergence of Klebsiella pneumoniae strains with reduced susceptibly against 3rd generation cephalosporins and carbapenems in Lagos hospitals, Nigeria.
}

\author{
Kabiru Akinyemi ${ }^{1}$, Rebecca Abegunrin ${ }^{1}$, Bamidele Iwalokun ${ }^{2}$, Christopher Fakorede ${ }^{1}$, \\ Oliwia Makarewicz ${ }^{3}$, Heinrich Neubauer ${ }^{4}$, Mathias Pletz ${ }^{3}$, and Gamal Wareth ${ }^{5}$ \\ ${ }^{1}$ Lagos State University \\ ${ }^{2}$ Nigerian Institute of Medical Research \\ ${ }^{3}$ Jena University Hospital \\ ${ }^{4}$ Friedrich-Loeffler-Institut Bundesforschungsinstitut für Tiergesundheit \\ ${ }^{5}$ Friedrich-Loeffler-Institut Bundesforschungsinstitut fur Tiergesundheit
}

September 14, 2020

\begin{abstract}
Different samples (i.e. stool, blood, urine, sputum, wound swabs, nasal swabs etc.) from 127 patients with suspected gramnegative infections based on on-site performed Gram-stain from four public hospitals between March and September 2015 in Lagos, Nigeria were analysed. Klebsiella (K.) pneumonia was identified in 43 (34\%) patients. Resistance rates of these 43 strains according to the CLSI breakpoints were as followed: cotrimoxazole (90.7\%), cefuroxime (74.4\%), ofloxacin (55.8\%), ceftazidime $(46.5 \%)$, and cefixime $(35 \%)$. Three isolates $(7 \%)$ were resistant to imipenem. All isolates were susceptible to amoxicillin/clavulanic acid and nitrofurantoin. The prevalence of ESBL-producing and carbapenem resistant strains (CRKP) was $69.8 \%$ and $7.0 \%$, respectively. Of the ESBL producing isolates, two K. pneumoniae isolates obtained from urine harbored both blaCTX-M-1 and blaSHV and a third isolate from urine harbored only blaCTX-M-1. Emerging of CRKP strains is an early warning signal for the prudent use of carbapenem antibiotics in concern to their efficacies.
\end{abstract}

\begin{abstract}
Different samples (i.e. stool, blood, urine, sputum, wound swabs, nasal swabs etc.) from 127 patients with suspected gram-negative infections based on on-site performed Gram-stain from four public hospitals between March and September 2015 in Lagos, Nigeria were analysed.Klebsiella $(K$.) pneumonia was identified in $43(34 \%)$ patients. Resistance rates of these 43 strains according to the CLSI breakpoints were as followed: cotrimoxazole $(90.7 \%)$, cefuroxime $(74.4 \%)$, ofloxacin $(55.8 \%)$, ceftazidime $(46.5 \%)$, and cefixime $(35 \%)$. Three isolates $(7 \%)$ were resistant to imipenem. All isolates were susceptible to amoxicillin/clavulanic acid and nitrofurantoin. The prevalence of ESBL-producing and carbapenem resistant strains (CRKP) was $69.8 \%$ and $7.0 \%$, respectively. Of the ESBL producing isolates, twoK. pneumoniae isolates obtained from urine harbored bothbla CTX-M-1 and bla SHV and a third isolate from urine harbored only bla CTX-M1. Emerging of CRKP strains is an early warning signal for the prudent use of carbapenem antibiotics in concern to their efficacies.
\end{abstract}

Keyword: Klebsiella pneumoniae ; Imipenem; ESBL; Emergence; Resistance; Nigeria.

\section{Introduction}

Klebsiella $(K$.)pneumoniae is a Gram-negative opportunistic nosocomial bacterial pathogen. It is involved 
in several localized and disseminated hospital-acquired infections such as burns infections, sepsis, respiratory and gastrointestinal tract infections, as well as urinary tract infections, pyogenic liver abscesses, soft tissue and wound infection (Ranjbar et al., 2019). The emergence of carbapenem-resistant K. pneumoniae (CRKP) strains has become an ultimate challenge for public health globally due to their ability to disseminate rapidly in the hospital environment and their extended antibiotic resistance phenotypes (Rossolini, 2015). The first CRKP isolate was reported from the USA in 2001 (Yigit et al., 2001). A few years later, outbreaks began to appear in several countries. Nowadays, it is the most common carbapenemase-producing Enterobacteriaceae $(\mathrm{CPE})$ and is considered one of the most rapidly growing global threats due to the high mortality in hospital-associated infections (Brink, 2019). In 2017, CRKP has been classified among those global critical pathogens listed by WHO concerning the discovery and development of new antibiotics.

In Nigeria, the prescription and use of carbapenem antibiotics is still low, and there is a lack of recent information on multidrug resistance (MDR)-associated K. pneumoniae. The present study was conducted to investigate the prevalence of $K$. pneumoniae among clinical samples of patients in four medical centers in Lagos, Nigeria, as well as to determine the burden of ESBL and carbapenemase-producing strains.

\section{Materials and methods}

The prevalence and burden of ESBL and CRKP strains were investigated involving 127 patients with various types of infections admitted to four public Lagos hospitals between March and September 2015. The Central Public Health Laboratory (CPHL), Lagos State University Teaching Hospital (LSUTH), National Institute of Medical Research (NIMR), and Iba Primary Health Centre (IPHC) contributed 42, 33, 32, and 20 samples, respectively. The institutional review boards of Lagos State University and the Nigerian Institute of Medical Research approved the study, and the consent of the patients was sought for. In total, 37 urine samples, 30 nasal swabs, and 20 wound swabs were aseptically collected from patients diagnosed with urinary tract infections, otitis media and pneumonia, and soft tissue abscess. Additionally, 20 fresh feces samples were collected from patients with gastroenteritis, and $4 \mathrm{ml}$ blood was collected from another 20 patients suffering from pyrexia of unknown origin (PUO). All blood samples from PUO patients were subjected to the thick-blood smear technique for the detection of the malaria parasite (Plasmodium spp.). Bacterial culture of all samples was done on brain heart infusion (BHI) and MacConkey agar (Oxoid, UK) and plates were incubated at $37^{\circ} \mathrm{C}$ overnight under aerobic conditions. The lactose fermenting discrete colonies were subjected to biochemical identification according to Cown and Steel 1993 (Phillips, 1993). Colonies biochemically confirmed as Klebsiella spp. were subjected to the MICROBACT 24E identification system (Oxoid, UK). All confirmed $K$. pneumoniae strains were tested against ten antibiotics by both, disc diffusion and microdilution methods according to the CLSI guidelines (CLSI, 2011). The following antibiotic discs were used: cotrimoxazole (COT) $(25 \mu \mathrm{g})$, ciprofloxacin (CIP) $(5 \mu \mathrm{g})$, ofloxacin (OFL) $(5 \mu \mathrm{g})$, gentamicin (GEN) $(10 \mu \mathrm{g})$, cefixime (CXM) $(5 \mu \mathrm{g})$, augmentin (AUG) (30 $\mu \mathrm{g})$, cefuroxime (CRX) (30 $\mu \mathrm{g})$, ceftazidime (CAZ) $(30 \mu \mathrm{g})$, imipenem (IMP) (30ug), nitrofurantoin (NIT) $(300 \mu \mathrm{g})$ (Oxoid, UK). The diameters of the zones of inhibition were measured with a ruler and compared with a zone-interpretation chart. The microdilution test was performed with the same set of antibiotics for consistency (Sigma, Deisenhofen, Germany) following the manufacturer's instructions. In this study, resistance was defined for isolates exhibiting intermediate resistance and resistance. Multidrug resistant (MDR) phenotype was defined as resistance to three or more classes of antibiotics.

All isolates that exhibited reduced susceptibility and resistance to third-generation cephalosporin were screened for ESBL production using Double Disc Synergy Test Method (DDST). This was done by placing the $3 \mathrm{GC}$ antibiotics i.e. ceftazidime $(30 \mu \mathrm{g})$ and ceftriaxone $(30 \mu \mathrm{g})$ at a distance of $15 \mathrm{~mm}$ (center to center) from $30 \mu \mathrm{g}$ augmentin, $20 \mu \mathrm{g}$ amoxicillin and $10 \mu \mathrm{g}$ clavulanic acid using CLSI interpretation guidelines (CLSI, 2011). Detection of carbapenemase enzyme activity was done following the modified Hodge test (MHT) as described by Landman and colleagues (Landman et al., 2005). For three ESBL gene makers, thebla CTX-M-1, bla SHV, and bla TEM PCR-based screening was conducted by PCR (Sturenburg et al., 2004, Pai et al., 1999).

\section{Results and discussion}


The prevalence of $K$. pneumoniae in the clinical samples was $34 \%(\mathrm{n}=43)$. K. pneumoniae was present in about half $(55.8 \%)$ of urine samples and a quarter $(23.3 \%)$ of nasal swabs. Three strains $(6.98 \%)$ were isolated from three patients with soft tissue infection, and three strains $(6.98 \%)$ were isolated from three cases of gastroenteritis (Table 1). Previous epidemiological investigations have shown that MDRK. pneumoniae strains harboring ESBL genes exist in environmental sources (Obasi et al., 2017) and are usually associated with nosocomial infections in Nigerian hospitals (Olalekan et al., 2019, Raji et al., 2013). However, there is still little data available on the prevalence of $K$. pneumoniae in Nigerian hospitals, and the published data are limited to regional studies and/or use of a limited number of samples (Hertz et al., 2019). Our results are comparable to the findings of Olalekan and colleagues, who identified K. pneumoniae in $35.4 \%$ of samples collected from four hospitals in Lagos between 2016-2018 (Olalekan et al., 2019), and Raji and co-workers, who found that K. pneumoniae represented $31.4 \%$ of strains collected during an assessment of the prevalence of drug resistance and ESBL among members of the family Enterobacteriaceae at Lagos teaching hospital (Raji et al., 2013).

In Nigeria, malaria is endemic. Three K. pneumoniae strains $(6.98 \%)$ were isolated from patients with concomitant Plasmodium falciparum infection. It can be speculated that $K$. pneumoniae -associated bacteraemia caused exacerbation of asymptomatic or silent malaria. Co-infections of Enterobacteriaceae with Plasmodium falciparum have been reported in some malaria-endemic African countries, including Nigeria, and were associated with increased severity (Akinyemi et al., 2007). Thus, such cases were expected also in this study. Hence, this is the first report on K. pneumoniae-Plasmodium spp . co-infections in patients within Lagos hospitals in recent times.

Most of isolates were resistant to cotrimoxazole (90.7\%), followed by cefuroxime (74.4\%), ofloxacin (55.8\%), ceftazidime (46.5\%), and cefixime (35\%). Three isolates $(7 \%)$ obtained from urine showed resistance to imipenem with a MIC $>8 \mathrm{mg} / \mathrm{L}$. Resistance rates were lowest for amoxicillin/clavulanic acid and nitrofurantoin $(0.0 \%)$ followed by gentamicin $(4.7 \%)$, imipenem $(7 \%)$, and ciprofloxacin $(28 \%)$. In total, $30 \mathrm{~K}$. pneumoniae isolates (69.8\%) were ESBL positive, of which 27 isolates (90\%) were multidrug-resistant MDR defined by resistance against three or more classes of antibiotics. (Table 2). In these MDR K. pneumoniae isolates eleven resistance patterns were identified and the pattern CAZ-CRX-OFL-CIP-COT [ceftazidime/cefuroxime/ofloxacin/ciprofloxacin/cotrimoxazole] was the most frequent one (Table 3). Two $K$. pneumoniae isolates from urine (U3 and U11) harbored both bla CTX-M-1 and bla SHV, and one isolate (U19) harbored only bla CTX-M-1. None of the tested strains contained the bla TEM. None of the three ESBL genes screened for was detected in the three carbapenem-resistant isolates. The emergence of resistance to imipenem is a new phenomenon and the explanation is inconclusive, because this drug is rarely used and relatively expensive. None of the three ESBL-genes was detected in any of the three carbapenemresistant $K$. pneumoniae isolates in our study. Only three genes have been checked by PCR due to limited resources, which is a limitation of this study.

Interestingly, the three CRKP strains were found in two different hospitals, at close distance of $20 \mathrm{~km}$. The emergence of ESBL due tobla CTX and bla SHV genes in K. pneumoniae has also been reported in other recent African studies: A high distribution of ESBL with the dominant bla CTX-M-1 gene marker was reported in Accra and Kumasi, Ghana (Falagas et al., 2011). A high prevalence of ESBL-producing K. pneumoniae in clinical isolates was reported in Côte d'Ivoire (Muller-Schulte et al., 2019). Strains harboringbla CTX-M-15, and bla SHV-134 were isolated from pig and abattoir workers in Cameroon (Founou et al., 2018).

In conclusion, this study revealed the emergence and dissemination of carbapenem resistant K. pnuemoniae isolates and bla CTX-M-1 and bla SHV co-harboring K. pneumoniae strains in Lagos. This study is limited by using a PCR screening tool that could only detect bla CTX-M-1, bla SHV and bla TEM. A comprehensive study on the real situation on carbapenemase-producing K. pneumoniae in Lagos using advanced molecular typing tools to assess the diversity and clonal relatedness of the strains is required to better monitor and understand the spread of MDR K. pnuemoniaein this region.

Acknowledgment: We are grateful to the staff of the Department of Microbiology and both the management of Lagos State University (LASU) and the Nigerian Institute of Medical Research (NIMR) for providing 
consumables and technical support respectively. Part of the abstract of this study was presented at the American Society for Tropical Medicine and Hygiene. It was published online in the American Journal of Tropical Medicine and Hygiene.

Funding: LASU and NIMR supported this work through provisions of consumables as well as through the joint efforts of the team.

Competing interests: None declared.

Authors' contributions: KOA conceived the study and designed the study protocol; ROA and COF carried out the literature search and antimicrobial susceptibility testing; KOA and BAI performed molecular analysis, organized, integrated all data and carried out the investigation. KOA, ROA, and GW interpretation of data and drafted the manuscript; KOA, OM, MWP, HN, and GW critically revised the manuscript for intellectual content. All authors read and approved the final manuscript. KOA is a guarantor of the paper.

\section{References}

Akinyemi, K. O., B. S. Bamiro and A. O. Coker, 2007: Salmonellosis in Lagos, Nigeria: incidence of Plasmodium falciparum-associated co-infection, patterns of antimicrobial resistance, and emergence of reduced susceptibility to fluoroquinolones. Journal of health, population, and nutrition, 25, 351-358.

Brink, A. J., 2019: Epidemiology of carbapenem-resistant Gram-negative infections globally. Current opinion in infectious diseases, 32, 609-616.

CLSI, 2011: Clinical and Laboratory Standards Institute. Performanc Standardsfor Antimicrobial Susceptibility Testing. Nineteenth Informational Supplement. CLSI document M100-S21. Wayne, PA, USA.

Falagas, M. E., D. E. Karageorgopoulos and P. Nordmann, 2011: Therapeutic options for infections with Enterobacteriaceae producing carbapenem-hydrolyzing enzymes. Future microbiology, 6, 653-666.

Founou, L. L., R. C. Founou, M. Allam, A. Ismail, C. F. Djoko and S. Y. Essack, 2018: Genome Sequencing of Extended-Spectrum beta-Lactamase (ESBL)-Producing Klebsiella pneumoniae Isolated from Pigs and Abattoir Workers in Cameroon. Frontiers in microbiology, 9, 188.

Hertz, F. B., F. Jansaker, K. O. Okon, I. S. Abdulmumin, J. O. Onah, J. Ladan and J. D. Knudsen, 2019: ESBL-production in Escherichia coli and Klebsiella pneumoniae isolates from Nigeria.MicrobiologyOpen, 8, e00816.

Landman, D., J. K. Salvani, S. Bratu and J. Quale, 2005: Evaluation of techniques for detection of carbapenem-resistant Klebsiella pneumoniae in stool surveillance cultures. Journal of clinical microbiology,43, 5639-5641.

Muller-Schulte, E., M. N. Tuo, C. Akoua-Koffi, F. Schaumburg and S. L. Becker, 2019: High prevalence of ESBL-producing Klebsiella pneumoniae in clinical samples from central Cote d'Ivoire.International journal of infectious diseases: IJID: official publication of the International Society for Infectious Diseases .

Obasi, A., S. Nwachukwu, E. Ugoji, C. Kohler, A. Gohler, V. Balau, Y. Pfeifer and I. Steinmetz, 2017: Extended-Spectrum beta-Lactamase-Producing Klebsiella pneumoniae from Pharmaceutical Wastewaters in South-Western Nigeria. Microbial drug resistance (Larchmont, N.Y.), 23, 1013-1018.

Olalekan, A., F. Onwugamba, B. Iwalokun, A. Mellmann, K. Becker and F. Schaumburg, 2019: High proportion of carbapenemase producing Escherichia coli and Klebsiella pneumonia e among extended spectrum beta-lactamase producers in Nigerian hospitals. Journal of global antimicrobial resistance .

Pai, H., S. Lyu, J. H. Lee, J. Kim, Y. Kwon, J. W. Kim and K. W. Choe, 1999: Survey of extended-spectrum beta-lactamases in clinical isolates of Escherichia coli and Klebsiella pneumoniae : prevalence of TEM-52 in Korea. Journal of clinical microbiology, 37, 1758-1763. 
Phillips, I., 1993: Cowan and Steel's Manual for the Identification of Medical Bacteria. J Clin Pathol, 46, 975-975.

Raji, M. A., W. Jamal, O. Ojemhen and V. O. Rotimi, 2013: Point-surveillance of antibiotic resistance in Enterobacteriaceaeisolates from patients in a Lagos Teaching Hospital, Nigeria. Journal of infection and public health, 6, 431-437.

Ranjbar, R., A. Fatahian Kelishadrokhi and M. Chehelgerdi, 2019: Molecular characterization, serotypes and phenotypic and genotypic evaluation of antibiotic resistance of the Klebsiella pneumoniaestrains isolated from different types of hospital-acquired infections.Infection and drug resistance, 12, 603-611.

Rossolini, G. M., 2015: Extensively drug-resistant carbapenemase-producing Enterobacteriaceae : an emerging challenge for clinicians and healthcare systems. Journal of internal medicine, 277, 528-531.

Sturenburg, E., A. Kuhn, D. Mack and R. Laufs, 2004: A novel extended-spectrum beta-lactamase CTXM-23 with a P167T substitution in the active-site omega loop associated with ceftazidime resistance.The Journal of antimicrobial chemotherapy, 54, 406-409.

Yigit, H., A. M. Queenan, G. J. Anderson, A. Domenech-Sanchez, J. W. Biddle, C. D. Steward, S. Alberti, K. Bush and F. C. Tenover, 2001: Novel carbapenem-hydrolyzing beta-lactamase, KPC-1, from a carbapenemresistant strain of Klebsiella pneumoniae. Antimicrobial agents and chemotherapy, 45, 1151-1161.

Table 1: Numbers and types of collected samples and $K$. pneumoniae positive isolates that have been used in the current study distributed in relation to the source of sampling.

\begin{tabular}{llllll}
\hline Types of samples & No. of collected samples (positive) & CPHL & LASUTH & NIMR & IPHC \\
\hline Urine & $37(24)$ & $9(6)$ & $10(5)$ & $12(8)$ & $6(5)$ \\
Nasal swab & $30(10)$ & $11(4)$ & $9(3)$ & $7(2)$ & $3(1)$ \\
Wound swab & $20(3)$ & $7(1)$ & $5(1)$ & $3(1)$ & $5(0)$ \\
Feces & $20(3)$ & $9(1)$ & $2(0)$ & $6(2)$ & $3(0)$ \\
Blood & $20(3)$ & $6(1)$ & $7(2)$ & $4(0)$ & $3(0)$ \\
TOTAL & $127(43)$ & $42(13)$ & $33(11)$ & $32(13)$ & $20(6)$ \\
\hline
\end{tabular}

The Central Public Health Laboratory (CPHL), Lagos State University Teaching Hospital (LSUTH), National Institute of Medical Research (NIMR), and Iba Primary Health Centre (IPHC)

Table 2: Distribution of $K$. pneumoniae ESBL, MDR, and CAB in relation to clinical samples.

\begin{tabular}{llllll}
\hline Samples & $\begin{array}{l}\text { Tested No. } \\
(\mathbf{\%})\end{array}$ & $\begin{array}{l}\text { Positive No. } \\
(\mathbf{\%})\end{array}$ & $\begin{array}{l}\text { ESBL+ No. } \\
\mathbf{( \% )}\end{array}$ & $\begin{array}{l}\text { MDR+ No. } \\
\mathbf{( \% )}\end{array}$ & $\begin{array}{l}\text { CAB+ No. } \\
(\mathbf{\%})\end{array}$ \\
\hline Urine & $37(29.2)$ & $24(55.8)$ & $16(66.7)$ & $17(70.8)$ & $3(12.5)$ \\
Nasal swab & $30(23.7)$ & $10(23.3)$ & $7(70.0)$ & $4(40.0)$ & $0(0.00)$ \\
Wound swab & $20(15.7)$ & $3(6.98)$ & $2(66.7)$ & $1(33.3)$ & $0(0.00)$ \\
Feces & $20(15.7)$ & $3(6.98)$ & $2(66.7)$ & $2(66.7)$ & $0(0.00)$ \\
Blood & $20(15.7)$ & $3(6.98)$ & $3(100)$ & $3(100)$ & $0(0.00)$ \\
TOTAL & $127(100)$ & $43(34 \%)$ & $30(69.8)$ & $27(62.8)$ & $3(6.98)$ \\
\hline
\end{tabular}

ESBL: Extended Spectrum ß-lactamase; MDR: Multidrug resistant; CAB: Carbapenem; $(+)$ : positive resistance

Table 3: Patterns of MDR to K. pneumoniae isolates from clinical samples 


\begin{tabular}{llll}
\hline Sample types & No. of isolates & Strains ID & Resistance pattern \\
\hline Urine and Blood & 5 & U12, U36, B37, U38, U42 & CRX-OFL-COT \\
Urine & 4 & U10, U23, U30, U43 & CAZ-CRX-OFL-COT \\
Feces & 1 & F31 & CXM-OFL-CIP-COT \\
Urine & 1 & U7 & CRX-CXM-GEN-OFL-COT \\
Urine & 2 & U3, U11 & CAZ-CRX-CXM-OFL-COT \\
Urine & 2 & U13, U35 & CAZ-CRX-CXM-IPM-COT \\
Blood & 1 & B40 & CRX-CXM-OFL-CIP-COT \\
Urine, Wound \& nasal swab & 3 & W17, U19, N26 & CAZ-CRX-OFL-CIP-COT-CXM \\
Urine & 1 & U18 & CAZ-CRX-OFL-CIP-COT-CXM-IPM \\
Urine \& nasal swab & 2 & U39, N4 & CAZ-OFL-CIP-COT \\
Urine, nasal swab, feces \& blood & 5 & B33, N9, N16, F32, U41 & CAZ-CRX-OFL-CIP-COT \\
Total & 27 & & \\
\hline
\end{tabular}

CAZ: Ceftazidime (30 $\mu \mathrm{g})$; CRX: Cefuroxine (30 $\mu \mathrm{g})$; GEN; Gentamicin $(10 \mu \mathrm{g})$; CXM: Cefixime $(5 \mu \mathrm{g})$;

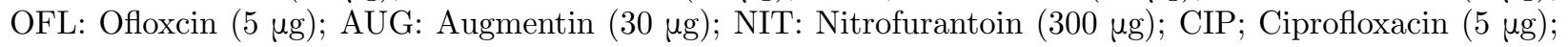
IPM: Imipenem (30 $\mu \mathrm{g})$; COT: Cotrimozaxole $(25 \mu \mathrm{g})$; U (urine), B (blood), F (feces), W (wound swab), N (nasal swab). 